\title{
Traditional Herbal Medicines - The Role of Polyphenols
}

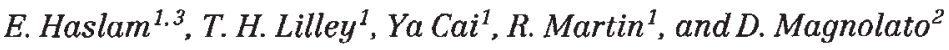 \\ ${ }^{1}$ Department of Chemistry, University of Sheffield, Sheffield S3 7HF, United Kingdom \\ ${ }^{2}$ Nestlé Research Department, Nestlé Ltd., Vers-Chez-Les-Blancs, CH-1000, Lausanne, Switzerland \\ ${ }^{3}$ Address for correspondence
}

Received: September 17, 1988

\section{Abstract}

The polyphenol composition of some traditional herbal remedies is reviewed. Polyphenols probably act in such remedies by virtue of their astringent action and current views on the molecular basis of this property are outlined and discussed.

\section{Introduction}

From the earliest of times man has been dependent on plants as sources of food and sustenance, for materials as diverse as paper for writing and wood for construction, and from particular plants he has derived substances ranging from perfumes, essences and spices to medicines and drugs. One of the earliest recorded uses of plants rich in polyphenols (tannins) was in the conversion of raw animal hides and skins to durable, non-putrescible leathers. A number of traditional plant-derived folk medicines are likewise rich in polyphenols (1). The outer-skin of the root of the tree paeony (Paeonia lactiflora), known as Mudan, is thus used medicinally to cure disorders of the bloodstream, including high blood pressure, and its usage dates back at least 2000 years. Its active principle is most probably the gallotannin (1); other examples of plants rich in polyphenols which find use as traditional medicines are shown in Table 2. In the constant effort to improve not only the efficacy but also, in oriental eyes, the ethics of modern medical practice researchers are increasingly turning their attention to folk medicines as sources of new drugs and to investigate the chem-

Table 1 Plant polyphenols: physiological and pharmacological properties.

\section{Foodstuffs and Beverages}

(a) Flavour, palatability and acceptability (Astringency)

(b) Nutritional Value

(c) Non-biological hazes, ageing of wines

(d) Herbal teas - aesophogeal cancer

(e) Toxic effects - molluscicidal action

2. Herbal medicines

(a) Effects on vascular system - blood pressure and capillary action

(b) Antiviral (antiherpetic) effects

(c) Stomach disorders, dysentry, diarrhoea, haemorrhages

(d) Microbial toxicity - antiseptic action

(e) Dressing of burns and inflammations

(f) Inhibition of direct acting mutagens

3. Chemical Defence in Plants

\section{Table 2 Some medicinal plants containing polyphenolic metabolites.}

1. Three Paeony (Paeonia lactiflora): outer skin of the root, used to cure disorders of the bloodstream, including high blood pressure. Principal polyphenolic metabolite the gallotannin (1).

2. Bearberry (Arctostaphylos uva-urs): dried leaves; infusions have a soothing astringent effect which have value as a diuretic, in kidney disorders and ailments of the bladder and urinary tract. Polyphenolic constituents have been described in detail by Haslam and Britton and include arbutin, various galloyl esters of arbutin and the gallotannin (1).

3. Agrimony (Agrimonia sp.): roots and dried aerial parts of the herb. Used as an astringent on the digestive system, a diuretic and as a haemostatic agent. The principal polyphenolic constituents include compounds (3-6).

4. Geranii Herba (Geranium maculatum, G. thunbergi): dried rhizome and leaves, employed as an astringent, anti-haemorrhagic and anti-inflammatory agent. Geraniin (7) is the principal crystalline polyphenol of Geranium species.

5. Meadowsweet (Filipendula ulmaria): aerial parts of plant - leaves and flowers - used as an infusion. Used as a mild astringent in digestive problems and as a diuretic. Its major polyphenolic constituent is rugosin-D (8).

6. Raspberry (Rubus idaeus): leaves and fruit. Raspberry leaf tea made from an infusion of dried leaves is used as a mouth gargle and for children's stomach disorders. It has a long tradition of use during pregnancy when taken regularly and liberally. The principal polyphenolic metabolite is sanguin $\mathrm{H}-6$ (9).

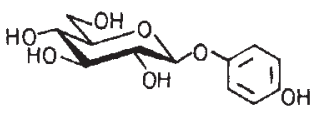

(2)

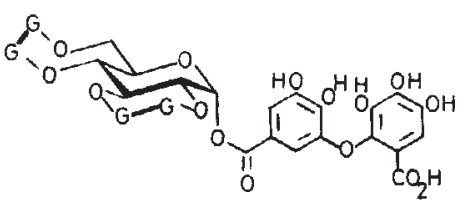

$(\stackrel{4}{\sim})$

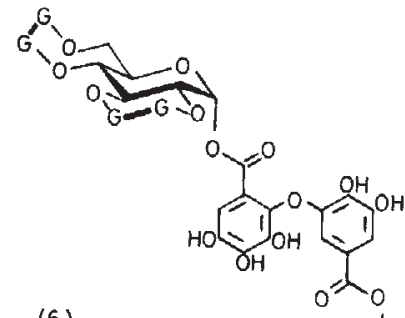

(6)

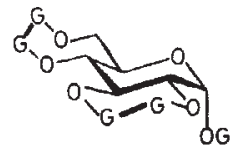

(3)

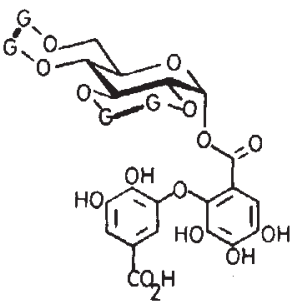

(5) 

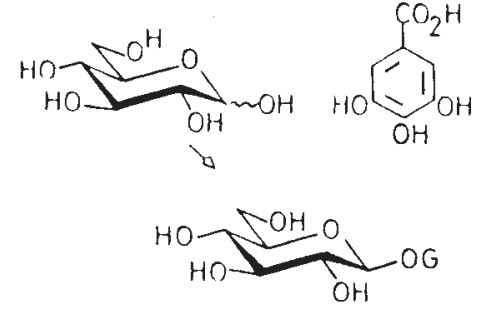

$y$
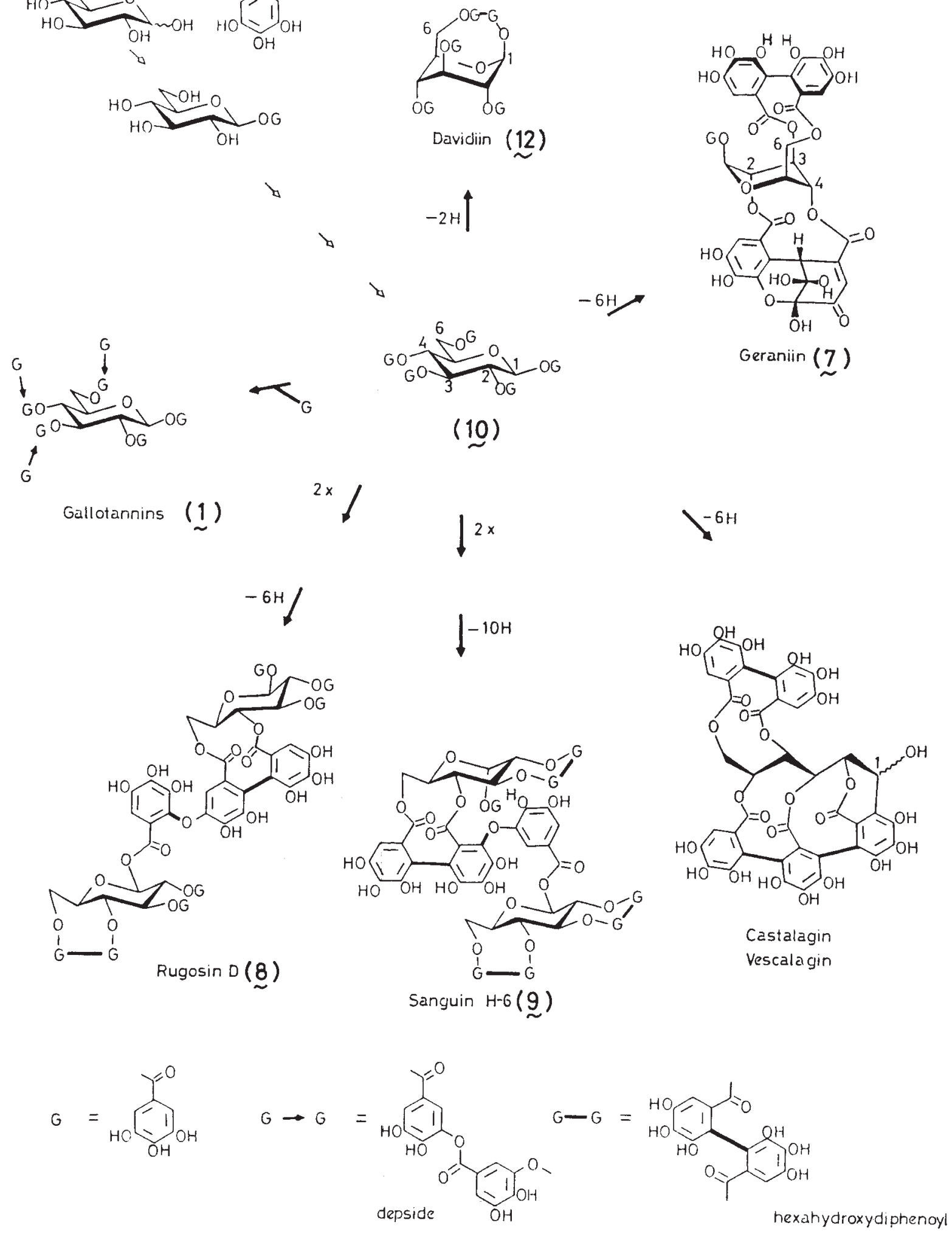

Davidiin (12)

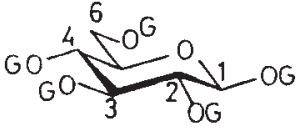

(10)

Fig. 1 Polyphenolic metabolites based on gallic acid.

(11) 
ical and biological basis of old remedies. Although the uses of polyphenols (tannins) as medicinal agents may be summarised under several headings (Table 1), many appear to devolve, at least in the first instance, on their astringent action. They thus aid, for example, the healing of wounds, burns and inflammation. In doing so the polyphenols act to produce an impervious layer [polyphenol-protein and/or polysaccharide complex] under which the natural healing processes can occur. Similar complexation processes probably take place internally; gut secretions are hindered thus protecting the underlying mucosa from toxins and other irritants in the bowels.

Studies of the reversible association of polyphenols with proteins have a long history. One of the first scientific papers on this topic was that of Sir Humphry Davy in 1803. This early work (2) demonstrated some of the macroscopic features of polyphenol complexation and it gave rise to several, wholely empirical, definitions of the term vegetable tannin. However until such times as structurally defined plant polyphenols became available the molecular mechanisms which underly polyphenol-protein complexation were not amenable to systematic investigation and were, in consequence, poorly understood. In the past $10-15$ years various studies of polyphenol complexation with both proteins and polysaccharides have been conducted (3-5). These investigations now permit a much more detailed picture of the molecular processes involved to be outlined.

\section{Discussion}

The association of polyphenols with proteins and polysaccharides is a specific example of the very important biological phenomenon of molecular recognition. The principles which underly molecular recognition may be analysed not only in terms of the composition, structure and conformation of both acceptor (host) and donor (guest) molecules but also in terms of three idealised concepts. "Dye-mould" ("jig-saw") matching is essentially static with an exact fit of donor and acceptor molecules. "Key-lock" matching is time dependent since the key (donor) invariably has to be manoeuvered into the lock (acceptor) to achieve the necessary precise fit. Finally "handin-glove" matching of donor and acceptor is both time-dependent and dynamic. Donor and acceptor molecules are mobile and flexible and may assume a variety of shapes as complexation proceeds. In such situations, to bring about strong and effective binding, it is imperative that many points of contact are ultimately established between donor and acceptor species. Such associative processes frequently exhibit strong cooperative effects. Present evidence strongly indicates that polyphenol complexations are largely of the "hand-in-glove" type. The critical questions which remain to be answered concern the nature and the identity of the points of matching and recognition with proteins and polysaccharides.

Contemporary studies of polyphenol complexation have been considerably facilitated by the availability for the first time of polyphenolic metabolites, particularly those based on gallic acid (Fig. 1), in a homogeneous state and with a range of very clearly defined molecular masses and conformations. The investigation of polyphenol-protein complexation has taken precedence probably because of its assumed greater importance and significance but also because of the ready access to proteins readily defined in terms of their molecular size and shape. Similar quantitative studies of polyphenol-car-

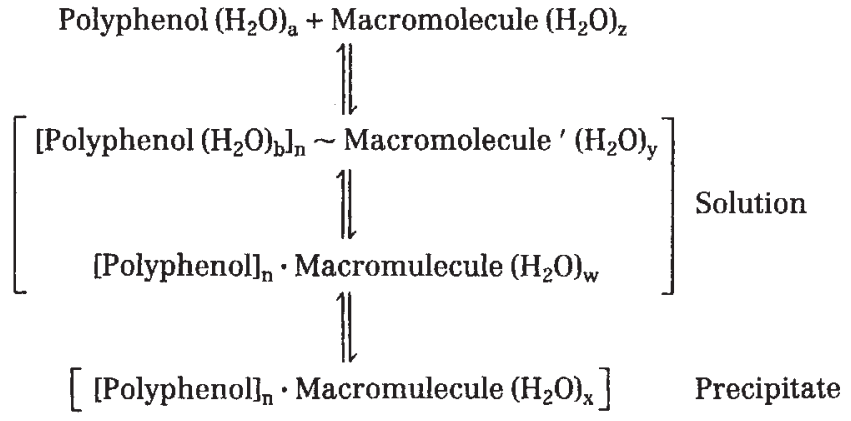

Fig. 2 Polyphenol complexation: reorganisation of water of solvation.

bohydrate complexation have been hampered to a large extent by the unavailability of water-soluble polysaccharides with similarly clearly defined molecular parameters of size and shape. Indeed the most satisfactory data have been obtained using polysaccharides in the solid phase - the chromatographic gels Sephadex G-10, G-25 and G-50, and LH-20, and cellulose acetate in membrane form (3).

One of the key factors in this whole problem of polyphenol complexation is the role of the solvent water (Fig. 2). As Szent-Gyorgi once cryptically remarked "Biology has forgotten water, or never discovered it".

The presence and influence of water is intuitively accepted but invariably tacitly ignored. The structure of the aqueous media surrounding or included within molecules such as proteins, polysaccharides and polyphenols is nevertheless one of the most critical facets of their structure. The degree of order of the solvent externally is generally proportional to its proximity to hydrophilic groups on the substrate molecular surface where water molecules are anchored by hydrogen bonding to acceptor and donor functionalities (e.g. $\mathrm{OH}, \mathrm{CO}$, CONH, etc.). During the complexation process the reorganisation of the various solvation shells provides an important driving force for the association of the polyphenol with the macromolecule, (Fig. 2). Given the presumed importance of these forces some detailed thought should be given in the future to the hydrophilic and hydrophobic effects associated with phenolic nuclei. Accepting these various riders the critical facets of

Table 3 Polyphenol-protein complexation (structure-activity relationships).

1. Complexation is dependent on protein type and $\mathrm{pH}$. Association is essentially a surface phenomenon, maximised at or near the isoelectric point of the protein and it occurs probably via the intermediacy of hydrogen bonding and hydrophobic interactions.

2. Proline-rich proteins (e.g. gelatin, salivary proteins) have a high affinity for polyphenols. Proteins which are small and tightly folded (e.g. myoglobin) have a relatively low affinity for polyphenols.

3. Molecular size of the polyphenol. In, for example, the galloyl-o-glucose series the efficacy of binding increases exponentially in the series tri $\rightarrow$ tetra $\rightarrow$ penta $\left(M_{\mathrm{R}}: 636,788\right.$ and 940$)$.

4. Conformational flexibility of the polyphenol. When conformational restraints are placed on the polyphenol substrate then its capacity to complex, whatever its molecular size, is dramatically reduced. Complimentarity leading to enhanced complexation between the polydentate ligand (polyphenol) and the receptor (protein) is maximised by conformational mobility in both components.

5. Water solubility of the polyphenol. A broadly inverse relationship exists between the strength of association and the solubility of the polyphenol in water. Low solubility favours strong association. 

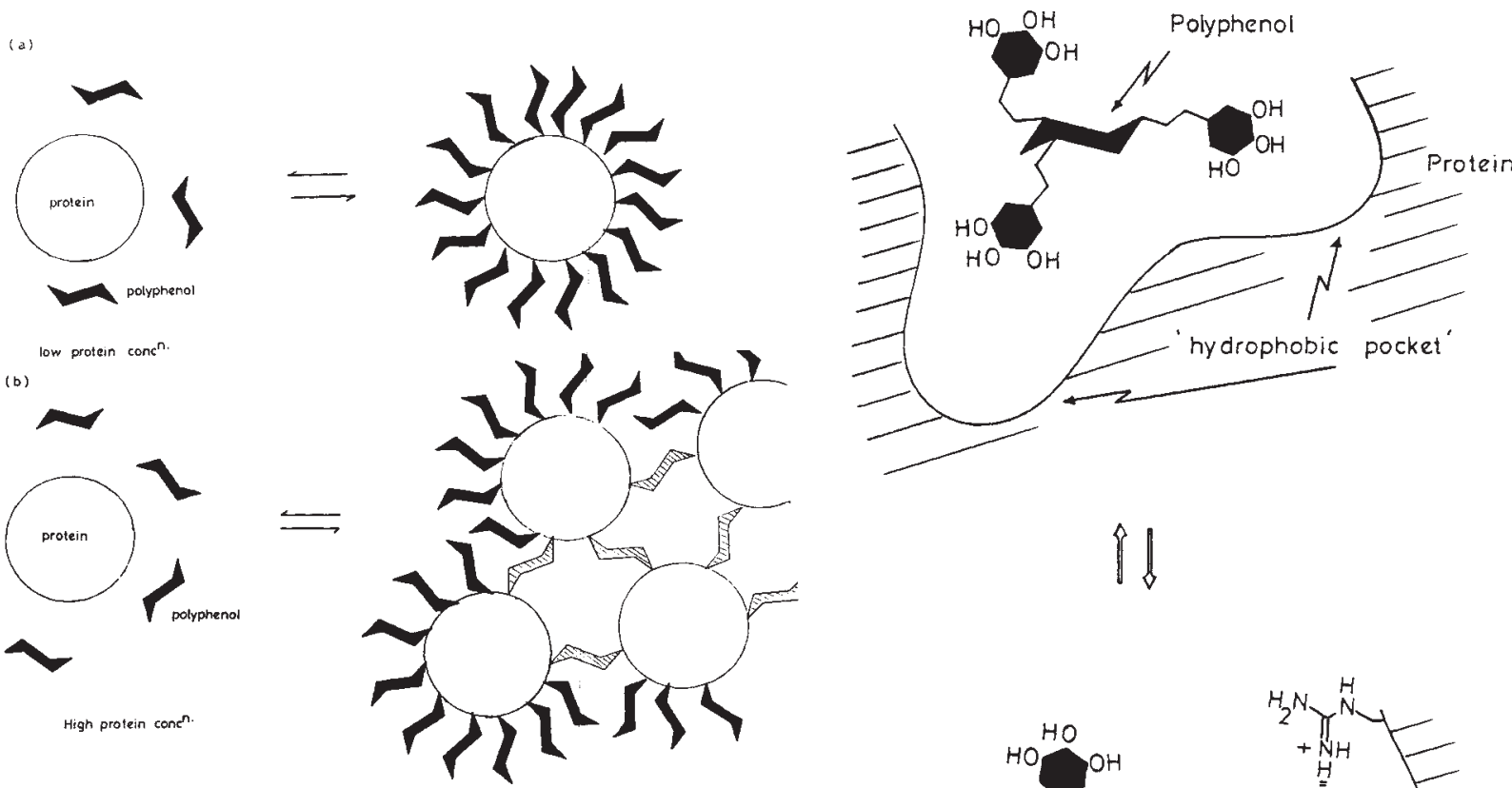

(c)

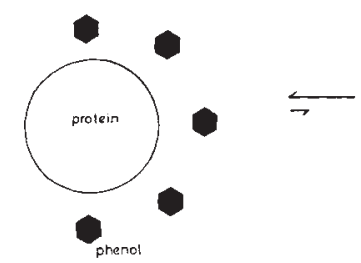

Fig. 3 Polyphenol-protein precipitation.

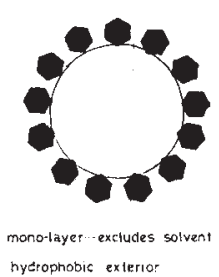

hystophobic exterior

Table 4 Polyphenol-polysaccharide complexation (structure-activity relationships).

1. Complexation is dependent on polysaccaride type but is broadly independent of $\mathrm{pH}(4-7)$. Open filamentous polysaccharides (1- $\alpha$-6-dextrans) bind polyphenols weakly. Polysaccharides which contain crevices, pores or holes in their three-dimensional structure or polysaccharides which assemble in solution loosely to form pores or holes within the structure bind polyphenols strongly. Association is principally mediated probably by sequestration of the polyphenol substrate into the pores.

2. Molecular size of the polyphenol. Increased molecular size leads to significantly enhanced affinity for polysaccharide.

3. Conformational flexibility of the polyphenol. When conformational restraints are placed on the polyphenol substrate then its capacity to complex is reduced. Overall shape and mobility is probably of as much significance in the polyphenol structure as the number of phenolic graups.

4. Water solubility of the polyphenol. A broadiy inverse relationship exists between the strength of association and the solubility of the polyphenol in water.

polyphenol complexation with proteins and polysaccharides are summarised in Tables 3 and 4.

Important features of polyphenol complexation which remain to be finally clarified concern the nature and the quantitative importance of the points of matching and recognition of host (polysaccharide, protein) and guest (polyphenol) molecules. Attention has focused primarily on (a)

hydrogen bonding and (b) hydrophobic effects as the mechanisms mediating complexation (6). Their relative significance remains a matter for continued speculation and debate but some attempts have been made to answer these questions, at least in a semi-quantitative manner.

The association of polyphenols with proteins is principally a surface phenomenon (Figs. 3a, b, c). The efficacy of polyphenol binding to protein derives from the fact that polyphenols are multidentate ligands able to bind sumultaneously (via different phenolic groups) at more than one point to the protein surface. When polyphenols cause precipitation of proteins from solution two situations may be envisaged. At low protein concentrations the polyphenol associates at one or more sites on the protein surface, to give a mono-layer which is less hydrophilic than the protein itself (Fig. 3a). Aggregation and precipitation then ensue. Where the protein concentration is high the relatively hydrophobic surface layer is formed by complexation of the polyphenol onto the protein and by crosslinking of different protein molecules by the multi-dentate polyphenols (Fig. 3b). Precipitation then follows as above. This tendency to cross-link protein molecules at higher protein concentrations explains the changing stoichiometry of the aggregates with changing protein concentrations - an observation first hinted at by Sir Humphry Davy. More polyphenol is thus required to precipitate proteins from dilute solution than from concentrated solutions. 
A significant corollary of this hypothesis is that various simple phenols such as pyrogallol should also be capable of precipitating proteins from solution if they can be maintained in solutions at concentrations sufficient to push the equilibrium in favour of the protein-phenol complex and thus form a hydrophobic layer of simple phenol molecules on the protein surface (Fig. 3c). For many simple phenols the limit is provided by their solubility in water but it can be achieved with, for example, bovine serum albumin (BSA, $3 \times 10^{-5}$ molal) and pyrogallol (1 molal). Thus, when comparatively simple phenolic substrates are present in solution they will also compete with polyphenols for sites on the protein molecules in the complexation processes. Simple phenols, depending on their effective concentration in solution, may therefore be expected to modify polyphenol-protein complexation. Complexes can be dissociated by the addition of further protein, hydrophobic solvent or hydrogen bond acceptor solvents (e.g. acetone), urea, poly-vinylpyrrolidone, polyethylene glycols, non-ionic detergents and nitrogen heterocycles such as caffeine. Complex formation is $\mathrm{pH}$ dependent; each protein has a distinctive $\mathrm{pH}$ optimum which usually lies at or very near to the isoelectric point of the particular protein. Where measurements have been possible the extent of complex formation rapidly declines as the $\mathrm{pH}$ of the medium is raised above 9.0. The inference generally drawn from all these observations is that polyphenols interact with protein by the formation of strong non-covalent bonds rather than by the elaboration of ionic or covalent linkages, (6).

The general, more detailed picture which is now beginning to emerge is that the association of polyphenols with proteins is largely a surface phenomenon and is possibly a process which takes place in two distinct phases (7). The first of these is that in which the polyphenol seeks out preferred sites and regions on the protein where its numerous aromatic rings are most readily accommodated by the development of hydrophobic interactions. This selectivity can be satisfied in the region of aromatic residues and, particularly, where prolyl residues in the polypeptide chain, by virtue of their effect on polypeptide conformation, bring several such aromatic groups or hydrocarbon side chains into close juxtaposition to form a hydrophobic pocket or environment [Fig. 4(i)]. Presumably during the formation of this pocket some significant solvent exclusion will occur. This first stage of association is then firmly reinforced by the appropriate deployment of hydrogen bonds between phenolic residues and polar groups (e.g. guanidino, amide and peptide, amino, hydroxyl and carboxyl groups) in the vicinity of the primary site of "docking" of the polyphenol to the polypeptide surface [Fig. 4(ii)]. Flexibility in both protein and polyphenol is presumably of considerable benefit during this second stage. The net effect is to produce a reversible complex between protein and polyphenol and one which leaves the surface of the protein "coated" with a much less hydrophilic layer of polyphenol molecules and which leads ultimately to aggregation and to precipitation. The efficacy of polyphenols in this complexation process derives from the fact that they act as polydentate ligands acting through several potential sites (phenolic residues) with the protein. The protein substrate is, likewise, potentially a multi-site acceptor molecule.

Investigations of aspects of this dynamic picture of polyphenol-protein association have been pursued at the more intimate molecular level employing various polyphenols (Fig. 1) and caffeine (13, as a peptide mimic) and the cyclodextrins $(\mathbf{1 4}$, as a model for hydrophobic crevices or pores on a macromolecular surface).
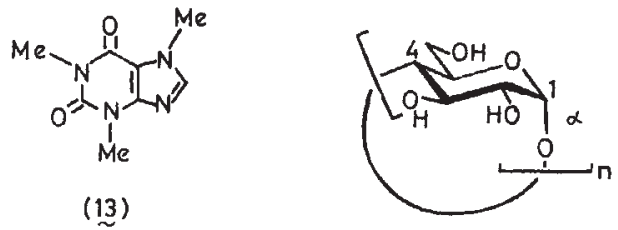<smiles>CC(=O)c1ccc(O)cc1</smiles>

$(14, n=6, \alpha$-cyclodextrin)

$(14, n=7, \beta-$ cycloclextrin $)$

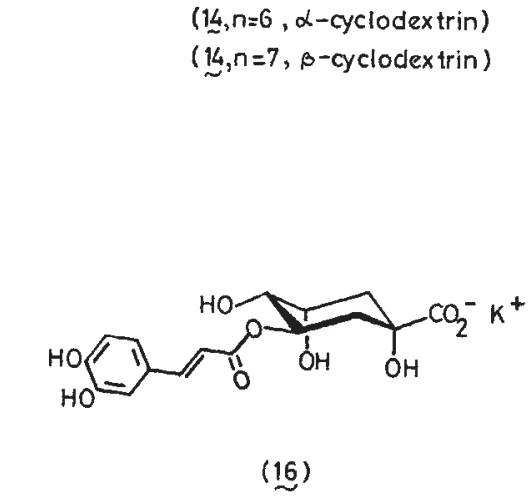

(16)

Whilst it is not possible to extrapolate directly to behaviour in solution, $\mathrm{X}$-ray crystallographic analysis of various caffeine-phenol complexes confirm the importance of (a) hydrogen bonding, (b) apolar hydrophobic interactions and, in certain situations, coordination around a metal ion as the primary intermolecular forces in caffeine-polyphenol complexation. In so far as the caffeine molecule (13) has structural elements which resemble a peptide-like structure, these observations probably also point to the significance of these same non-covalent forces in the association of polyphenols with proteins.

X-ray crystallographic analysis of complexes of caffeine (13) with a variety of phenolic substrates show that in the solid state a layer-lattice structure is frequently present. In this array caffeine and aromatic molecules are arranged in stacks in alternating layers, approximately parallel, with an interplanar separation of approx. 3.3 to $3.4 \AA$. For methyl gallate (15) this stacking structure is complemented by an extensive inplane system of hydrogen bonding between the three phenolic hydroxyl groups of methyl gallate and the two ketoamide groups and the basic N-9 of caffeine (8). The crystal structure of the caffeine complex with potassium chlorogenate (16) first isolated from coffee beans by Gorter shows similar features to those noted with methyl gallate (9). However, an additional critical stabilising factor is the coordination of seven oxygen atoms in an irregular polyhedral arrangement around the central potassium ion $\left(\mathrm{K}^{+} \ldots\right.$ O distances between 2.67 and $2.85 \AA$ ). A further feature of interest in these crystalline phenol-caffeine complexes is the relative orientation of the planar caffeine and phenolic partner in the layer lattice.

The association of caffeine with various polyphenols has also been studied by microcalorimetry and by ${ }^{1} \mathrm{H}$-NMR spectroscopy (7). The latter technique exploits the tendency of phenolic molecules and caffeine to "stack" (vide supra) in solution. This in turn results in a deshielding of the aromatic protons of the phenol and of the three methyl groups and the single proton of the caffeine molecule and this causes upfield shifts in the respective ${ }^{1} \mathrm{H}-\mathrm{NMR}$ signals. The ${ }^{1} \mathrm{H}-\mathrm{NMR}$ spectrum of caffeine is composed of four singlets. One from each of the 
three methyl groups and one from the proton attached to carbon atom (C-8).Various workers have observed that, at fixed caffeine concentration, the addition of aromatic substrates to the caffeine solution causes an "upfield" shift of each of the four singlets due to magnetic anisotropic effects. This property may be exploited to calculate the equilibrium constant for the association between caffeine and the aromatic substrate. Use has been made of equation 1, an analogue of the Benesi-Hildebrand equation, and also making due allowances for the self-dimerisation of both caffeine and the aromatic substrate:

$$
\frac{1}{\Delta}=\frac{1}{\mathrm{~K} \cdot \Delta_{0}^{\mathrm{AP}}} \frac{1}{\left[\mathrm{P}_{0}\right]}+\frac{1}{\Delta_{0}^{\mathrm{AP}}}
$$

where: $\Delta=$ Chemical shift change induced by substrate $P$ at concentration $\left[\mathrm{P}_{0}\right]$,

$\left[\mathrm{P}_{0}\right]=$ Formal substrate concentration,

$\mathrm{K}=$ Equilibrium constant for the formation of $1: 1$ complex between caffeine and the substrate $P$,

$\Delta_{0}^{\mathrm{AP}} \quad=$ Chemical shift difference between the caffeine resonance in the unbound state and the state in which it is totally in the form of a $1: 1$ complex.

The equilibrium constants for the formation of a $1: 1$ complex between caffeine (13) and a series of natural polyphenols have been determined at various temperatures (Table 5). Significantly, these results show exactly the same trends which were observed earlier (3) in studies of polyphenol-protein complexation, namely a very strong dependence on molecular size of the polyphenol and on its conformational flexibility. They thus strongly confirm and reinforce the view, originally assumed, that polyphenol-caffeine complexation not only mirrors in many of its facets polyphenol-protein complexation, but is also a good model system with which to examine the general mechanisms of association. Particularly striking are the depressive effects on complexation which re-

Table 5 Polyphenol: caffeine complexation. Association constants $\left(K_{A P}\right.$ molar $^{-1}$ litre) in deuterium oxide.

\begin{tabular}{|c|c|c|c|}
\hline & & $60^{\circ} \mathrm{C}$ & $45^{\circ} \mathrm{C}$ \\
\hline \multirow[t]{13}{*}{ A. } & Galloyl Esters & & \\
\hline & Methyl gallate (15) & 11.0 & - \\
\hline & Davidin (12) & 23.2 & - \\
\hline & Corilagin (28) & 16.7 & - \\
\hline & Casuarictin (27) & 18.9 & - \\
\hline & Tellimagrandin 1 & 21.9 & - \\
\hline & $\beta-1,3,6$-Tri-O-galloyl-D-glucose & 36.4 & - \\
\hline & $\beta-1,2,6$-Tri-O-galloyl-D-glucose & 42.1 & - \\
\hline & Eugeniin (26) & 61.9 & - \\
\hline & $\beta$-1,2,4,6-Tetra-O-galloyl-D-glucose & 52.3 & - \\
\hline & $\beta$-Penta- $O$-galloyl-D-glucose $(10)$ & 97.9 & - \\
\hline & Sanguin $\mathrm{H}-6(9)$ & 71.5 & - \\
\hline & Rugosin-D (8) & 135.6 & - \\
\hline \multirow[t]{5}{*}{ B. } & Flavan-3-ol-Derivatives & & \\
\hline & (+)-3-O-Galloylcatechin (19) & 26.8 & 38.3 \\
\hline & (-)-3-O-Galloylepigallocatechin (23) & 35.1 & 52.8 \\
\hline & Procyanidin B-2 (25) & - & 26.1 \\
\hline & Procyanidin B-3 (24) & - & 22.3 \\
\hline
\end{tabular}

Association constants quoted as average of four values abtained by calculation respectively from the chemical shift changes $(\Delta \delta)$ of the proton at C-8 and the three $\mathrm{N}$-methyl groups of caffeine in $\mathrm{D}_{2} \mathrm{O}$, using an external standard.
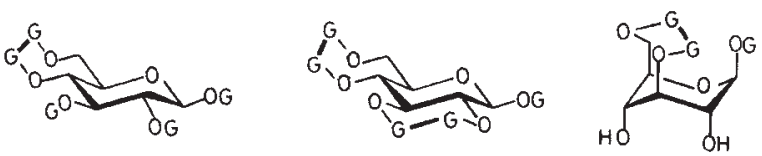

(26, Eugeniin)

$(27$, Casuarictin)

(28)<smiles>[R]Oc1ccc(C2Oc3cccc(O)c3C[C@H]2O)cc1</smiles>

(17, $R=H$ )

$(18, \mathrm{R}=\mathrm{OH})$<smiles>O=C(O[C@H]1Cc2c(O)cccc2O[C@H]1c1ccc(O)cc1)c1cccc(O)c1</smiles>

(23)<smiles>O=C(CC1Cc2ccccc2OC1c1ccccc1)c1ccccc1</smiles>

(19)<smiles></smiles>

$\left(\underline{20}, R^{1}=R^{2}=H\right)$

$\left(22, R^{1}=R^{2}=O H\right)$
(21, $R^{\prime}=\mathrm{OH} ; \mathrm{R}^{2}=\mathrm{H}$ )

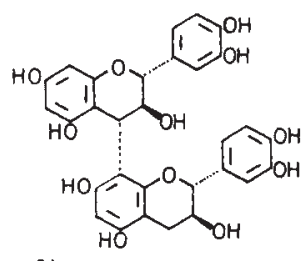

(24, Procyanidin 8-3)

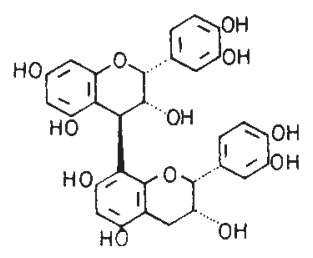

$(25$, Procyanidin B-2)

sult from restriction of conformational mobility by the biosynthetic formation of the hexahydroxydiphenoyl ester group (11) in the polyphenolic substrate (Table 5), the strong temperature dependence of complexation (Table 5) indicative of significant entropy effects (which perhaps suggests important solvation effects), and the relatively weaker affinity of typical procyanidins $(24,25)$ as compared to galloyl esters for caffeine (Table 5). This latter observation underlines the generally assumed weaker affinity which proanthocyanidins (condensed tannins) have for proteins when compared to galloyl esters (hydrolysable tannins).

Studies of polyphenol-polysaccharide association indicates (vide supra, Table 4) that the ability of the polysaccharide host to generate shapes which are able to encapsulate, either wholely or in part, the polyphenol guest is often a critical feature which promotes strong binding. In order to study this form of hydrophobic interaction and to comprehend its significance in protein-polyphenol complexation more fully attention has been directed towards the interaction of polyphenols with $\alpha$ - and $\beta$-cyclodextrins.

Cyclodextrins, first isolated in 1891, are cyclic oligosaccharides composed of (1- $\alpha-4)$-linked $D$-glucosyl residues; $\alpha$ - and $\beta$-cyclodextrins possess respectively 6 and 7 glucose units. They have the shape of a doughnut with all the $\mathrm{D}$ glucopyranose units in substantially undistorted $\left({ }^{4} \mathrm{C}_{1}, \mathrm{C}-1\right)$ conformations. The cavities are slightly "V" shaped; the secondary hydroxyl groups (at C-2 and C-3) on the upper side of the torus 


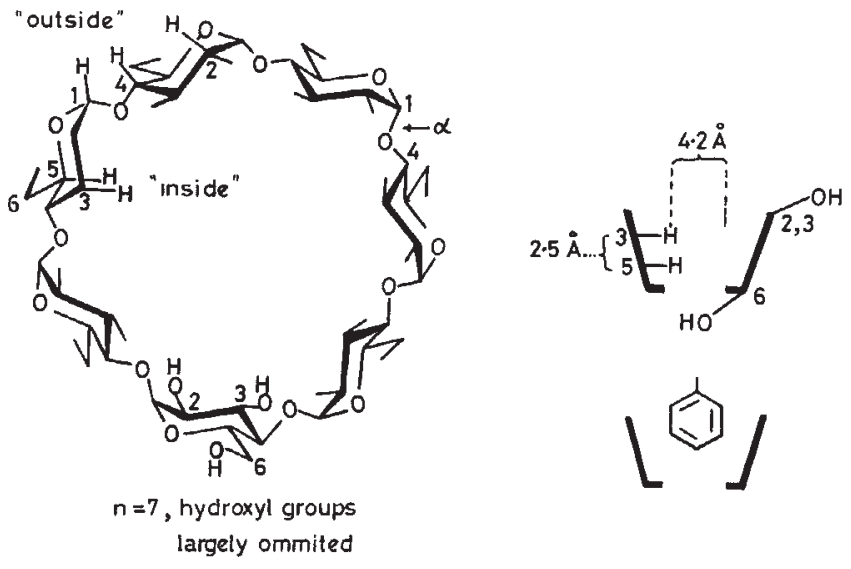

Fig. $5 \quad \beta$-Cyclodextrin.

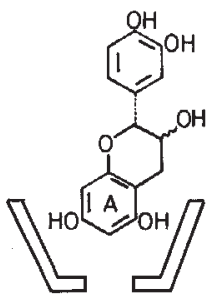

(a)

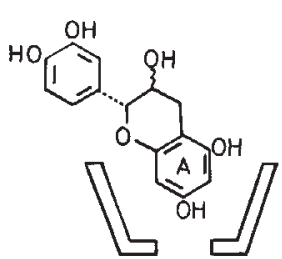

(b)

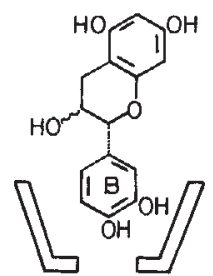

(c)
Fig. 6 Polyphenol- $\beta$-cyclodextrin sequestration.

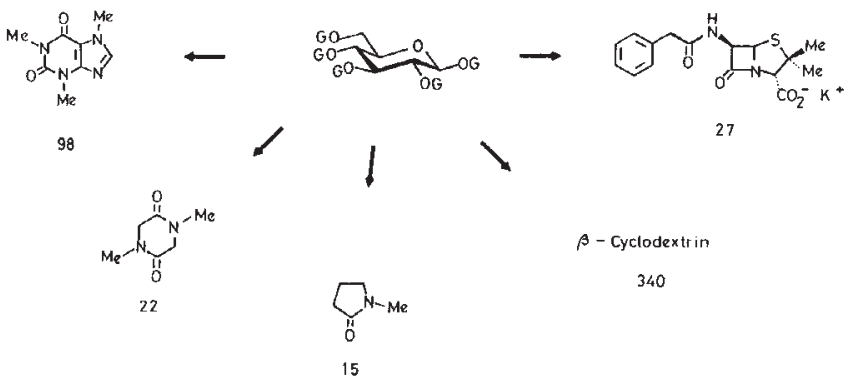

Fig. 7 Association constants for complexation reactions of $\beta$-pentagalloyl-Dglucose (10).

$$
\text { ASSOCIATION CONSTANTS (mole litre) }-45^{\circ} \mathrm{C}-\mathrm{D}_{2} \mathrm{O}
$$

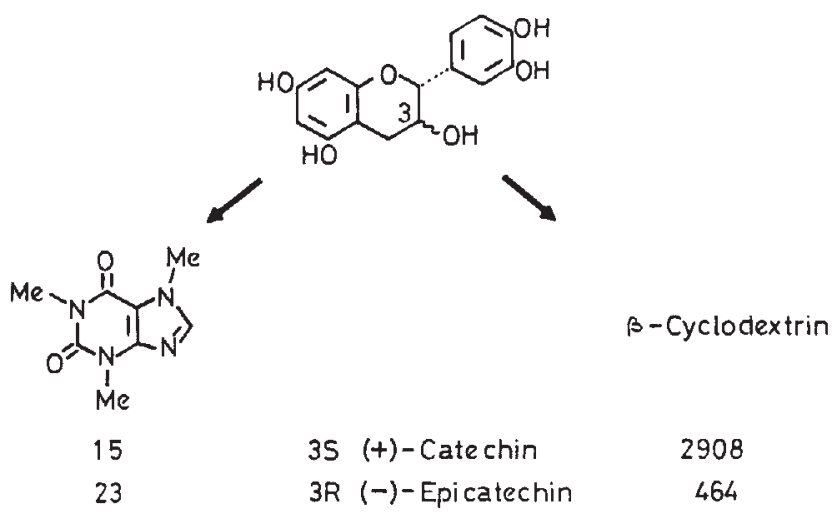

Fig. 8 Association constants for complexation reactions of flavan-3-ols. and the primary hydroxyl groups (at C-6) on the lower face. The interior of the torus consists of the glycosidic oxygen atoms and two concentric rings of $\mathrm{C}-\mathrm{H}$ groups (at $\mathrm{C}-3$ and $\mathrm{C}-5$, Fig. 5). The cavity is generally thought to be more accessible from the face bearing the secondary hydroxyl groups and, compared to an aqueous environment, is apolar and relatively hydrophobic.

One of the most important properties of the cyclodextrins is their ability to sequester substrates in the hydrophobic cavity. The nature of the binding and the driving forces which lead to inclusion remain uncertain although several suggestions have been put forward (10) including (a) hydrogen bonding, (b) van der Waals interactions, (c) release of strain energy in the cyclodextrin, and (d) release of solvent water molecule(s) from the cavity. In order to ascertain the importance of "cavity sequestration" in relation to polyphenol complexation overall, the mechanism of the formation of cyclodextrin inclusion complexes with phenolic substrates has been investigated by high-resolution ${ }^{1} \mathrm{H}$ - and ${ }^{13} \mathrm{C}$-NMR spectroscopy. The resonances of $\mathrm{H}-3$ and $\mathrm{H}-5$, located inside the cavity of the cyclodextrins, show substantial changes of chemical shift $(\delta)$ upon complexation with aromatic compounds, including polyphenols. This provides direct and compelling evidence for the insertion of the aromatic nucleus of the phenolic substrate into the cyclodextrin cavity in aqueous media. The ${ }^{1} \mathrm{H}$ - and ${ }^{13} \mathrm{C}-\mathrm{NMR}$ signals of the phenolic guest also display characteristic chemical shift changes and measurement of these various complexation-induced shifts and application of variations of the Benesi-Hildebrand equation leads to the association constant for the formation of the inclusion complex. Measurements were made for various phenols with $\alpha$ - and $\beta$ cyclodextrin (Table 6). A number of features command immediate attention. Compared to galloyl esters the binding of phenolic flavan-3-ols is quite strong. It is dependent on the stereochemistry of the hydroxyl group at C- 3 in the flavan and is diminished by hydroxyl substitution in the B-ring. Complexation is enhanced by galloylation at the 3 hydroxyl group: modestly $(2 \times)$ in the case of $(+)$-catechin $(\mathbf{1 7})$, but substantially $(9 \times)$

Table 6 Polyphenol: cyciodextrin complexation. Association constants $\left(\mathrm{K}_{\mathrm{CP}}\right.$ molar $^{-1}$ litre) in deuterium oxide.

\begin{tabular}{l|rc}
\hline & \multicolumn{1}{c}{$\boldsymbol{\beta}$} & $\alpha$ \\
\hline Flavan-3-ols(318 K) & & \\
(+)-Catechin (17) & 2908 & - \\
(+)-Catechin 3-gallate (19) & 6232 & - \\
(+)-Gallocatechin (18) & 948 & - \\
(-)-Epiafzelechin (20) & 792 & - \\
(-)-Epicatechin (21) & 464 & - \\
(-)-Epigallocatechin (22) & 208 & - \\
(-)-Epigallocatechin 3-gallate (23) & 1889 & - \\
Procyanidin B-3 (24) & 101 & - \\
Procyanidin B-2 (25) & 63 & - \\
Miscellaneous phenols (318 Ka or 298 K $\left.\mathrm{K}^{\mathrm{b}}\right)$ & & \\
p-Hydroxybenzoic acid & $652^{\mathrm{a}}$ & $472^{\mathrm{b}}$ \\
3,4-Dihydroxybenzoic acid & $459^{\mathrm{a}}$ & $702^{\mathrm{b}}$ \\
Methyl 3,4-dihydroxybenzoate & $280^{\mathrm{a}}$ & $138^{\mathrm{a}}$ \\
Gallic acid & $114^{\mathrm{b}}$ & $215^{\mathrm{b}}$ \\
Methyl gallate & $147^{\mathrm{b}}$ & - \\
Methyl 2,3,4-trihydroxybenzoate & $212^{\mathrm{a}}$ & - \\
Resorcinol & $117^{\mathrm{b}}$ & \\
Phloroglucinol & $110^{\mathrm{b}}$ & \\
$\beta$-1,2,3,4, 6-Penta-O-galloyl--g-glucose (10) & 340 (at 333 K)
\end{tabular}

Association constants quoted as average of several measurements calculated from the chemical shift changes $(\triangle \delta)$ of $\mathrm{H}-3$ and $\mathrm{H}-5$ of the cyclodextrin. 
with (-)-epigallocatechin (22). Significantly substitution at C-4 by additional flavan-3-ol molecules to give the typical pro(antho)cyanidin structures $(24,25)$ virtually suppresses completely the ability to complex with the cyclodextrins.

In the case of polyphenol : cyclodextrin complexation a wide range of parameters has been probed and measured in an attempt to throw light on the manner in which the phenolic substrates associate with the oligosaccharide host. For more complex phenolic substrates, as for example the phenolic flavan-3-ols and their derivatives (17-25) the expectation is that several modes of association are probably in operation involving the insertion of both rings $A$ and $B$ into the cyclodextrin cavity. (Figs. 6a, b, c). Clearly, although a great deal more work remains to be carried out to produce a wholely satisfying rationale of the manner of polyphenolic complexation with cyclodextrins, several important features have emerged. Polyphenolic substrates have a strong affinity for preformed oligosaccharide "holes" such as are provided by the cyclodextrins. Complexation is probably largely of the "lock and key" type and is critically dependent on the size of the cavity and structural and stereochemical features of the polyphenolic substrate. It is nevertheless evident that natural polyphenols have a peculiar affinity for "holes, pores or crevices" in polysaccharide structures whether these are preformed (cyclodextrins and Sephadex gels) or whether they develap as the polyphenol and oligosaccharide undergo molecular recognition (amyloses). Finally it is interesting to note, particularly in respect of the model of polyphenol-protein complexation presented earlier (Fig. 4), the comparative strengths of association of some representative polyphenols with both $\beta$-cyclodextrin and caffeine (Figs. 7 and 8). These observations strongly suggest that hydrophobic effects are probably of similar if not greater significance, particularly in the "early" stages of molecular recognition, as hydrogen bonding in bringing about the complexation of polyphenols with proteins. Further work is in progress on this problem and on its relationship to the mode of action of polyphenols in herbal medicines.

\section{Acknowledgements}

The authors wish to thank the Agriculture and Food Research Council (S. H. G.), Nestlé Company Limited (Switzerland) R. M., and the Government of the Peoples' Republic of China (Y.C.) for financial support, and Professor I. Nishioka (Kyushu University, Japan) for the gift of a sample of (-)-epiafzelechin.

\section{References}

${ }^{1}$ Haslam, E., Lilley, T. H. (1988) CRC Rev. Food Sci. Nutrition, 27, 1 40.

2 White, T. (1957) J. Sci. Food Agric. 8, 377.

3 McManus, J. P., David, K. G., Beart, J. E., Gaffney, S. H., Lilley, T. H., Haslam, E. (1985) J. Chem. Soc., Perkin Trans 2, 1429.

${ }^{4}$ Hagermann, A. E., Butler, L. G. (1981) J. Biol. Chem. 256, 4491.

5 Oh, H. I., Hoff, J. E., Armstrong, G. S., Haff, L. A. (1980) J. Agric. Food Chem. 28, 394.

${ }^{6}$ Loomis, W. D. (1974) Methods Enzymol. 31, 528.

7 Spencer, C. M., Cai, Y., Martin, R., Gaffney, S. H., Goulding, P. N., Magnolato, D., Lilley, T. H., Haslam, E. (1988) Phytochemistry 27, 2397-2409.

8 Martin, R., Lilley, T. H., Bailey, N. A., Falshaw, C. P., Haslam, E., Magnolato, D., and Begley, M. J. (1986) J. Chem. Soc., Chem. Commun. 105-106.

9 Martin, R., Falshaw, C. P., Haslam, E., Lilley, T. H., Magnolato, D., Begley, M. J. (1987) Phytochemistry 26, 2733.

10 Bender, M., Komiyama, K. (1978) Cyclodextrin Chemistry, Reactivity and Structure Concepts in Organic Chemistry, 6, Springer Verlag, Basel. 